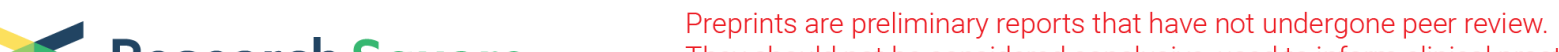 Research Square They should not be considered conclusive, used to inform clinical practice, or referenced by the media as validated information.
}

\section{Effect of direct angioplasty therapy on acute middle cerebral artery occlusion with good leptomeningeal collateral}

\section{Ying Luo}

Affiliated Hospital of Southwest Medical University

Jun Huang

The Third Hospital of Mianyang

\section{Yang Yang}

Affiliated Hospital of Southwest Medical University

Hong Liu

Affiliated Hospital of Southwest University

\section{Xiu Chen}

Affiliated Hospital of Southwest Medical University

\section{Xiaogang Li}

Affiliated Hospital of Southwest Medical University

\section{Zuoxiao Li}

Affiliated Hospital of Southwest Medical University

\section{Bo Liu}

The Third Hospital of Mianyang

Jinglun Li ( $\nabla$ xnykdx1951@163.com )

Affiliated Hospital of Southwest Medical University

\section{Research article}

Keywords: acute middle cerebral artery occlusion, good leptomeningeal collateral, recanalization, angioplasty

Posted Date: December 5th, 2019

DOl: https://doi.org/10.21203/rs.2.18228/v1

License: (c) (1) This work is licensed under a Creative Commons Attribution 4.0 International License.

Read Full License 


\section{Abstract}

Background: The objective of this study was to investigate and discuss the effect of direct angioplasty therapy on acute middle cerebral artery occlusion (MCAO) patients with good leptomeningeal collateral circulation in 4.5 hours. Methods: We retrospectively reviewed our acute ischemic stroke database from January, 2017 to January, 2019, then selected consecutive patients with evidence of the proximal M1 segments of MCAO and good leptomeningeal collateral who have received angioplasty or mechanical thrombectomy (MT). The baseline characteristics and outcome of patients was statistical analysis, included age, gender and risk factors, baseline national institutes of health stroke scale (NIHSS) scores, preoperative alberta stroke programme early CT (ASPECT) score, time from door to needle, time of door to puncture, endovascular procedure time, 7d NIHSS score and the modified treatment in cerebral infarction ( $\mathrm{m}-\mathrm{TICl}) 2 \mathrm{~b}$ or 3 , symptomatic hemorrhage, average hospital stays, modified rankin scale (mRS) score 02 at 3-month and mortality. All the thrombi were analyzed by histopathology. All statistical analysis was done with $t$ test for continuous data and $\chi 2$ test for binary data. Results: A total of 93 patients were included (direct angioplasty $=41(44.1 \%)$, MT $=52(55.9 \%))$. There was no significant difference in baseline data between the two groups. The difference in the time of door to recanalization, the time of puncture to recanalization, symptomatic hemorrhage, and average hospital stays were significantly different between groups $(P<0.05)$. The other agents were not significantly different between groups ( $P>0.05$ each). Histopathological analysis showed all thrombi contained different amounts of platelets, fibrinogen, white blood cell, and red blood cell. Conclusion: Direct angioplasty therapy on acute MCAO with good leptomeningeal collateral may help to shorten the time of surgery, reduce symptomatic hemorrhage, and hospital stay.

\section{Background}

Mechanical thrombectomy (MT) can significantly improve the prognosis of patients with acute large vessel occlusion stroke (LVOS) and reduce disability and mortality [1]. However, in the real world, the good prognosis rate after MT is still low. A recent study has shown that its substantial reperfusion in $85.9 \%$, adverse events were symptomatic intracranial hemorrhage $(\mathrm{sICH})$ in $6.7 \%$ of patients, and at discharge, only $23.0 \%$ had functional independence (mRS 0-2) [2]. There is a mismatch between reperfusion of blood flow after MT and good prognosis. Whether it is a time window or a tissue window, shortening the time of blood flow recanalization and reducing operative cerebral hemorrhage are the most beneficial factors for improving the prognosis of acute ischemic stroke (AIS) $[3,4]$.

The optimal recanalization treatment, postoperative complications, and prognosis of acute vessels occlusion due to cardioembolic or atherosclerosis-related in situ stenosis/occlusion may be completely different [5]. Studies have shown that compared with cardiac embolism (CE), for large vascular occlusion caused by large artery atherosclerosis (LAA), arterial thrombectomy is more likely to cause serious complications such as symptomatic cerebral hemorrhage [6]. Previous study has shown that the pros and cons of collateral circulation after cerebral artery occlusion may be a potential identification method [7]. Cerebral collateral circulation refers to the blood flow through other blood vessels countercurrent 
when the cerebral vascular disease causes blood flow to decrease or disappear, providing blood perfusion to the ischemic brain tissue, so that the ischemic tissue receives different degrees of perfusion compensation [8]. Among them, the leptomeningeal collateral circulation provides bidirectional blood supply to the cerebral cortex, which is an important collateral pathway in the acute middle cerebral artery occlusion (MCAO) [9]. The collateral circulation of CE is poorly compensated, while the collateral circulation of the in-situ thrombus is well compensated [10]. Angioplasty has proven to be safe and effective for artery stenosis or occlusion on account of LAA $[11,12]$.

A latest systematic review and meta-analysis found that atherosclerosis-related occlusions have a longer rate of puncture to reperfusion and a higher percentage of balloon angioplasty or intracranial stenting is performed to achieve successful revascularization [13]. Then, for the AIS probably caused by LAA in the hyperacute phase, is it feasible to directly perform angioplasty therapy? Therefore, this study aimed to explore the safety and efficacy of direct angioplasty to acute MCAO with good leptomeningeal collateral.

\section{Methods}

\section{Patients}

From January, 2017 to January, 2019, consecutive patients with AIS who have received endovascular therapy (ET) and met the criteria for inclusion were retrospectively reviewed: 1) $\geq 18$ years; 2) acute neurological symptoms within 4.5 hours; 3 ) computerized tomography (CT) scan excluded intracranial hemorrhagic; 4) clinical diagnosis of AIS [1]; 5) a pre-treatment national institutes of health stroke scale (NIHSS) score of $\geq 9 ; 6$ ) a pre-stroke modified Rankin Scale score (mRS) between $0-1 ; 7)$ no contraindications for intravenous thrombolysis; 8) no hypersensitivity to iodinated contrast media; 9) the M1 segments of MCAO confirmed by digital substraction angiography (DSA); 10) with good leptomeningeal collateral circulation, ASTIN/SIR [14] 2-4; 11) a consent form for revascularization was signed [23].

Intravenous thrombolytic treatment with recombinant tissue plasminogen activator (rtPA) (Germany Boehringer Ingelheim Pharmaceutical, Co., Ltd,) at a dose of $0.9 \mathrm{mg} / \mathrm{kg}$. First, $10 \%$ of the intravenous bolus was given within 5 minutes, and the rest was intravenously pumped within one hour.

Patients were evaluated based on baseline characteristics, including age, gender, vascular risk factors, $\mathrm{mRS}$, NIHSS score, and reperfusion therapy related variables. After diagnostic cerebral angiography, different revascularization methods were performed according to random number table. Group A were treated with direct angioplasty, the others (group B) were treated with MT.

\section{Endovascular procedures}

Sustained intravenous sedation at a rate of $1 \mathrm{ug} / \mathrm{kg}(4 \mathrm{ug} / \mathrm{ml})$ (Dexmedetomidine Hydrochloride Injection, Grand Pharmaceutical Co Ltd, China). The modified seldinger technique was used to puncture the right 
femoral artery and place the 8F catheter sheath (Stryker, Fremont, California). The iodixanol (270 mg $\mathrm{I} / \mathrm{mL}$, lodixanol Injection, GE Healthcare Co Ltd, Ireland) was injected at an injection rate of $5 \mathrm{~mL} / \mathrm{s}$, in a total amount of $10 \mathrm{ml}$ into CCA, $3 \mathrm{~mL} / \mathrm{s}$ and $6 \mathrm{ml}$ in VA. The catheter was continuously flushed with pressurized heparinized saline (5000 U/liter) to prevent thrombosis.

\section{Direct angioplasty}

The 0.014-inch micro-guidewire (Boston Scientific, Natick, Massachusetts) and microcatheter (Ev3 Inc., Irvine, California) were carefully fed into the $\mathrm{M} 3$ segment through an $8 \mathrm{~F}$ guiding catheter (Boston Scientific, Natick, Massachusetts). The micro-guidewire was withdrawn, and the microcatheter angiography showed smooth blood flow at the distal end and revealed the distal end of the vessel occlusion. Reinserting the micro-guidewire and withdrawing the microcatheter, an appropriately sized balloon dilatation catheter (Gateway, Boston Scientific, Natick, Massachusetts) was delivered to the distal end of the occlusion, and the position of the balloon was determined by angiography and then pressurized (6-12 atm) by a pressure based on the condition of vasodilation. After the stenosis was significantly relieved, if the blood flow can be maintained $\mathrm{m}-\mathrm{TICl}$ grade of $2 \mathrm{~b}$ or 3 for 10 minutes, the operation was terminated, otherwise a neuroform EZ (Boston Scientific, Natick, Massachusetts) stent will be placed in the vascular lesion. No subsequently remedial procedures were performed.

\section{MT (stent retrievers and/or contact aspiration)}

A microcatheter (Ev3 Inc., Irvine, California) was positioned approximately $10 \mathrm{~mm}$ distal to the thrombus with a micro-guidewire (Ev3 Inc., Irvine, California) and an intracranial support catheter (Ev3 Inc., Irvine, California) was navigated to the proximal thrombus. The $6 \mathrm{~mm}$ * $30 \mathrm{~mm}$ retriever stent (Ev3 Inc., Irvine, California) was subsequently advanced to the distal tip of the microcatheter and the microcatheter was withdrawn to release the stent. After five minutes, the stent was pulled back into the microcatheter, and then both the microcatheter and the stent were withdrawn. An angiogram was used to evaluate the recanalization status. The entire procedures were repeated up to 3 times to achieve successful reperfusion defined as an $\mathrm{m}-\mathrm{TICl}$ grade of $2 \mathrm{~b}$ or 3 . If the retrievable stent thrombectomy did not remove the thrombus, a direct aspiration technique was used to remedial MT. The aspiration catheter (Penumbra Inc., Alameda, California) with ADAPT [15] technique was used to aspirating thrombus. No other subsequently remedial procedures were performed [23].

\section{Main Outcome Measures}

All patients were received CT scans immediately after operation, and 24 hours after the procedure to investigate possible location and size of cerebral infarction or hemorrhagic complications. Symptomatic Intracranial hemorrhage (ICH) was assessed on post-treatment CT scans and classified as hemorrhagic infarction or parenchymal hemorrhage based on the European cooperative acute stroke study III (ECASS III) criteria [16]. Assessment of angiographic images was performed by the consensus of two neuroradiologists who were blinded to the procedure. Neurological evaluation was performed by two 
stroke neurologists immediately after treatment, 24 hours, and 90 days after treatment. Functional outcomes at 90 days were assessed with a clinical visit. The mRS score $\leq 2$ was defined as good outcome. All the thrombi were analyzed by histopathology.

\section{Statistical Analysis}

For patient characteristics and outcomes, differences between groups were estimated by $t$ test for continuous data and $\chi 2$ test for binary data. All statistical analysis was done with statistical package for social science (SPSS) version 25.0 (IBM Corp., Armonk, NY), with $\mathrm{Cl}$ set at $95 \%$ and significant level set at 0.05 [23].

\section{Results}

Of 93 patients with AIS and evidence of the proximal M1 segments of MCAO who have received vascular recanalization from January, 2017 to January, 2019, direct angioplasty was performed in group $A(n=$ $41)$, while patients in group $B(n=52)$ have received MT. Comparison of baseline characteristics of patients from group A (angioplasty) and group B (MT) are provided in Table 1. All the factors were not significantly different between two groups $(P>0.05$ each) (Table 1$)$.

Comparison of outcome of patients from group A and group B are provided in Table 2. The time from onset to door, time of door to puncture, baseline NIHSS score, preoperative ASPECT score, $\mathrm{m}-\mathrm{TICl} 2 \mathrm{~b}$ or 3 , 7d NIHSS score, mRS score 0-2 at 3-month, symptomatic hemorrhage and mortality were not significantly different between groups $(P>0.05$ each) (Table 2$)$. The difference in the time of door to recanalization, the time of puncture to recanalization, symptomatic hemorrhage, and average hospital stays were significantly different between groups $(P<0.05)$.

Histopathological analysis showed all thrombi contained different amounts of platelets, fibrinogen, white blood cell, and red blood cell.

\section{Discussion}

This study found that compared with MT, as to the patients with acute MCAO and good leptomeningeal collateral circulation within 4.5 hours, direct angioplasty therapy can shorten the recanalization time, length of hospital stay, may reduce symptomatic cerebral hemorrhage, and did not weaken blood flow reperfusion and prognosis. And the leptomeningeal collateral circulation might be help to estimate the cause of acute MCAO. If the lateral branch is compensated well, the cause of occlusion may be an in-situ thrombus formed by atherosclerotic stenosis.

Result shows that patients with good leptomeningeal collateral circulation have more frequent hypertension, hypercholesterolemia and smoking, who are also the cause of LAA. Analysis of thrombus components had similar results. Due to the gradual slow formation of atherosclerosis in acute LVOS caused by LAA, the blood supply may be insufficient for a long period of time, making the collateral circulation relatively complete [17]. Therefore, it is more tolerant to ischemia after total occlusion, with 
milder symptoms, longer treatment window, and a lower risk of bleeding after opening [18]. On the contrary, CE-induced occlusion occurs more urgently, collateral circulation is relatively inadequate, ischemic tolerance is relatively poor, treatment time window is short, and risk of hemorrhagic transformation after vascular recanalization is higher [19].

The incidence of vascular wall injury and bleeding risk is related to different pathogenesis. Studies have shown that patients with LAA are more likely to have complications of cerebral hemorrhage after MT [6]. As to in-situ thrombosis occurring on the basis of severe stenosis caused by atherosclerosis, it is intertwined with fibrin and platelets, and is tightly bound to the vessel wall. The thrombectomy procedure can easily damage the wall of the vessel. Therefore, it is necessary to start antithrombotic treatment as soon as possible, and use arterial thrombolysis and angioplasty as the main method, otherwise the lesions may have repeated formation and restenosis of fresh blood vessels. On the contrary embolism is an acute thrombus shedding. The thrombus is mainly wrapped by red blood cells and does not adhere to the arterial wall. It is the best indication for MT [20].

Surgery-related results showed that the angioplasty group had shorter operative time and hospital stays, and less symptomatic hemorrhage. It may be because the operation of angioplasty has a longer history, less difficult and complications than MT. Cerebral collateral circulation has been described to play a significant role in preserving tissue viability in large vessel occlusion stroke (LVOS) with a favorable impact on time window for individualized treatment, angiographic reperfusion, complications and functional outcomes. Rebello et al [10] have discussed the potential role of collateral flow in stroke patients with AF versus stroke patients with LAA. For patients with intracranial anterior circulation LVOS caused, the results show that collateral scores were lower in the AF group with patients having poor collaterals in $28 \%$ of cases versus $9 \%$ in the LAA group. Mortality rates were higher in the AF patients whilst rates of any parenchymal hemorrhage were higher in the LAA group. Moreover, the proportion of large blood vessel occlusion caused by LAA in Asian population is $30 \% \sim 50 \%$, which is higher than $8 \% \sim 10 \%$ of white people $[21,22]$. This may be one of the reasons for the good effect of direct angioplasty.

The shortcomings of this study are that, it is difficult to accurately judge whether it is atherosclerosis or embolism. The sample size is small, and the results may be biased. More questions need to be answered. For example, what type of recanalization should be used for LAA with poor collateral circulation? Further large randomized studies are needed.

\section{Conclusion}

Direct angioplasty therapy on acute MCAO with good leptomeningeal collateral may help to shorten the time of surgery, reduce symptomatic hemorrhage and hospital stay. Further large randomized studies are needed.

\section{List Of Abbreviations}


NIHSS: National Institutes of Health Stroke Scale;

ASPECTS: Alberta Stroke Program Early CT Score;

m-TICl: modified thrombolysis in cerebral infarction;

mRS: modified rankin score;

MCAO: middle cerebral artery occlusion;

MT: mechanical thrombectomy;

LVOS: large vessel occlusion stroke;

DPT: door-to-puncture;

sICH: symptomatic intracranial hemorrhage;

AIS: acute ischemic stroke;

rtPA: recombinant tissue plasminogen activator;

CM: contrast media;

$\mathrm{ICH}$ : Intracranial hemorrhage;

SPSS: Statistical Package for Social Science;

LAA: large artery atherosclerotic infarction;

CE: cardiac embolism.

\section{Declarations}

\section{Ethics approval and consent to participate}

All procedures performed in the studies involving human participants were in accordance with the ethical standards of the institutional and/or national research committee and with the 1964 Helsinki Declaration and its later amendments or comparable ethical standards.

This study complies with national guidelines, 2018 Guidelines for the Early Management of Patients with Acute Ischemic Stroke, and local ethics committee rules.

\section{Consent for publication}

Written informed consent for publication was obtained from all participants. 
Availability of data and materials

The datasets generated and/or analysed during the current study are not publicly available due patients required not to disclose specific data, and they are available from the corresponding author on reasonable request.

\section{Competing Interests}

The authors declare that they have no conflict of interest.

\section{Informed consent}

Informed consent was obtained from all individual participants included in the study.

\section{Funding}

No funding was received for this study.

\section{Authors' contributions}

$\mathrm{JL}$ and $\mathrm{BL}$ conceived and designed the study. $\mathrm{YL}, \mathrm{JH}, \mathrm{BL}$ and $\mathrm{JLL}$ performed the experiments. $\mathrm{YL}$ and $\mathrm{JH}$ wrote the paper. YY, HL, XC, XGL and ZXL reviewed and edited the manuscript. All authors read and approved the manuscript.

\section{Acknowledgements}

Not applicable.

\section{References}

1.Powers WJ, Rabinstein AA, Ackerson T, Adeoye OM, Bambakidis NC, Becker K, et al; American Heart Association Stroke Council. 2018 Guidelines for the early management of patients with acute ischemic stroke: a guideline for healthcare professionals from the American Heart Association/American Stroke Association. Stroke. 2018;49: e46-e110.

2.Jahan R, Saver JL, Schwamm LH, et al. Association Between Time to Treatment with Endovascular Reperfusion Therapy and Outcomes in Patients with Acute Ischemic Stroke Treated in Clinical Practice[J]. JAMA, 2019,322(3):252-263.

3. O'Neill D, Griffin E, Doyle KM, et al. A Standardized Aspiration-First Approach for Thrombectomy to Increase Speed and Improve Recanalization Rates[J]. AJNR. American journal of neuroradiology, 2019.DOI:10.3174/ajnr. A6117.

4.Alawieh A, Vargas J, Fargen KM, et al. Impact of Procedure Time on Outcomes of Thrombectomy for Stroke[J]. Journal of the American College of Cardiology,2019,73(8):879-890.

5. HORIE N, TATEISHI Y, MORIKAWA M, MOROFUJI Y, HAYASHI K, IZUMO T, et al. Acute stroke with major intracranial vessel occlusion: characteristics of cardio-embolism and atherosclerosis-related in situ stenosis/occlusion[J]. J Clin Neurosci, 2016, 32:24-29]. 
6. Heo J H, Lee K Y, Kim S H, et al. Immediate reocclusion following a successful thrombolysis in acute stroke: A pilot study[J]. Neurology, 2003, 60(10):1684-1687.

7. Jia B, Liebeskind D S, Song L, et al. Performance of computed tomography angiography to determine anterograde and collateral blood flow status in patients with symptomatic middle cerebral artery stenosis[J]. Interventional Neuroradiology, 2017:159101991769448.

8. Liebeskind DS. Collateral circulation. Stroke, 2003,34:2279-84.

9. van Seeters T, Biessels GJ, Kappelle LJ, et al. Determinants of leptomeningeal collateral flow in stroke patients with a middle cerebral artery occlusion[J]. Neuroradiology, 2016,58(10):969-977.

10. Rebello LC, Bouslama M, Haussen DC, et al. Stroke etiology and collaterals: atheroembolic strokes have greater collateral recruitment than cardioembolic strokes[J]. European journal of neurology,2017,24(6):762-767.

11. Levy E I, Siddiqui A H, Crumlish A, et al. First Food and Drug Administration-Approved Prospective Trial of Primary Intracranial Stenting for Acute Stroke[J]. Stroke; a journal of cerebral circulation, 2009, 40(11):3552-3556.

12. Wang Y, Ma Y, Gao P, et al. Primary Angioplasty without Stenting for Symptomatic, High-Grade Intracranial Stenosis with Poor Circulation[J]. American Journal of Neuroradiology, 2018 Aug;39(8):14871492.

13. Tsang ACO, Orru E, Klostranec JM, et al. Thrombectomy Outcomes of Intracranial AtherosclerosisRelated Occlusions[J]. Stroke; a journal of cerebral circulation,2019,50(6):1460-1466.

14. Interventional TA, T. Neuroradiology, trial design and reporting standards for intraarterial cerebral thrombolysis for acute ischemic stroke[J]. Journal of Vascular\& Interventional Radiology, 2003, 14 (8): E1-E31.

15. TURK, A.S, FREI, D., FIORELLA, D., et al. ADAPT FAST study: A direct aspiration first pass technique for acute stroke thrombectomy[J]. Journal of neurointerventional surgery,2014,4(4):260-264.

16. Hacke W, Kaste M, Bluhmki E, Brozman M, Dávalos A, Guidetti D, et al; ECASS Investigators. Thrombolysis with alteplase 3 to 4.5 hours after acute ischemic stroke. N Engl J Med. 2008; 359:13171329.

17. Ramakrishnan G, Dong B, Todd KG, et al. Transient Aortic Occlusion Augments Collateral Blood Flow and Reduces Mortality During Severe Ischemia due to Proximal Middle Cerebral Artery Occlusion[J]. Translational stroke research,2016,7(2):149-155.

18. KIM H J, YUN S C, CHO K H, CHO A H, KWON S U, KIM J S, et al. Differential patterns of evolution in acute middle cerebral artery infarction with perfusion-diffusion mismatch: atherosclerotic vs. cardioembolic occlusion[J]. J Neurol Sci, 2008, 273 (1/2) :93-98.

19.HAO Y, YANG D, WANG H, ZI W, ZHANG M, GENG Y, et al; ACTUAL Investigators (Endovascular Treatment for Acute Anterior Circulation Ischemic Stroke Registry). Predictors for symptomatic intracranial hemorrhage after endovascular treatment of acute ischemic stroke[J]. Stroke, 2017, 48:12031209.

20. SPORNS P B, HANNING U, SCHWINDT W, VELASCO A, MINNERUP J, ZOUBI T, et al. Ischemic stroke: what does the histological composition tell us about the origin of the thrombus? [J]. Stroke, 2017, 48:2206-2210. 
21. WONG L K. Global burden of intracranial atherosclerosis[J]. Int J Stroke, 2006, 1:158-159.

22. WHITE H, BODEN-ALBALA B, WANG C, ELKIND M S, RUNDEK T, WRIGHT C B, et al. Ischemic stroke subtype incidence among whites, blacks, and Hispanics: the Northern Manhattan Study[J]. Circulation, $2005,111: 1327-1331$. This is one of the reasons why the effect of angioplasty was better in the study.

23. Luo Y, Yang Y, Xie Y, et al. Therapeutic effect of pre-operative tirofiban on patients with acute ischemic stroke with mechanical thrombectomy within 6-24 hours[J]. Interventional neuroradiology, 2019;25(6):705-709. 